\section{Tuberöse Sklerose}

Die Europäische Kommission hat die Zulassung erteilt für den oral verfügbaren mTOR-Inhibitor Everolimus (Votubia) zur Behandlung von erwachsenen Patienten mit renalem Angiomyolipom (AML), assoziiert mit einer tuberösen Sklerose (TSC), bei denen ein Risiko für Komplikationen vorliegt, die jedoch nicht unmittelbar operiert werden müssen. Novartis

\section{Morbus Parkinson}

Das Unternehmen Actavis hat Ropinirol als Retardtabletten zur täglichen Einmalgabe (Ropinirol-Actavis ${ }^{\circledR}$ ) in sein Portfolio aufgenommen. Der Dopaminagonist ist bei Parkinson als Monotherapie zur Initialbehandlung indiziert, um den Einsatz von L-Dopa hinauszuzögern. Außerdem wird Ropinirol in Kombination mit L-Dopa im weiteren Verlauf eingesetzt, wenn die Wirksamkeit von L-Dopa nachlässt oder unzuverlässig wird und Wirkschwankungen auftreten. RopinirolActavis $^{\circledR}$ ist in den Wirkstärken $2 \mathrm{mg}$, $4 \mathrm{mg}$ und $8 \mathrm{mg}$ erhältlich. Actavis

\section{Alzheimer-Demenz}

Die Zukunft des anti-beta-AmyloidMedikaments Solanezumab gegen Alzheimer-Demenz ist weiterhin offen. Am 8. Oktober wurden im Rahmen der Jahrestagung der American Neurological Association in Boston erstmals Daten der beiden Phase-III-Studien zu Solanezumab öffentlich vorgestellt. Die primären Endpunkte der Studien bei Patienten mit leichter bis mittelschwerer Alzheimer-Demenz wurden nicht erreicht. In einer vorspezifizierten Sekundäranalyse der gepoolten Daten aller Patienten mit leichter AlzheimerDemenz aus beiden Studien konnte jedoch eine signifikante Verlangsamung der Abnahme der kognitiven Fähigkeiten gezeigt werden. Dieses Ergebnis entspricht einer Verlangsamung der Abnahme der kognitiven Fähigkeiten um $34 \%$. Über das weitere Vorgehen wird nach Gesprächen mit den Zulassungsbehörden entschieden.

Restless-Legs-Syndrom

\title{
Transdermaler Dopaminagonist bewährt sich in der Praxis
}

Bei Patienten mit mittelgradigem bis schwerem Restless-Legs-Syndrom (RLS) wird in der Regel eine Therapie mit Dopaminagonisten empfohlen. In placebokontrollierten Studien hat sich hier die transdermale Applikation von Rotigotin (Neupro ${ }^{\circledR}$ ) bewährt. Darauf hat Professor Karin Stiasny-Kolster, Marburg, hingewiesen. So ließen sich mit Rotigotin als Pflaster (3 mg/24 h) in einer Studie mit 458 Patienten die RLS-Symptome auf der internationalen RLS-Skala IRLS um knapp 17 Punkte reduzieren (von 28 bis auf 11 Punkte), mit Placebo dagegen nur um 8,6 Punkte. In einer Auswertung von Daten über fünf Jahre kam es über den gesamten Zeitraum hinweg sowohl nachts als auch tagsüber zu keiner Verstärkung der Beschwerden.

Bestätigt werden die Ergebnisse von placebokontrollierten Studien nun auch durch die Praxisstudie RLS-PRACTISE, deren Resultate Stiasny-Kolster vorstellte. Teilnehmer waren 687 Patienten, die neu auf eine Therapie mit Rotigotin eingestellt wurden (im Schnitt 2,7 mg/d nach drei Monaten). Die meisten wurden auf das transdermale Präparat eingestellt, weil sie mit der Wirksamkeit der bisherigen Medikation nicht zufrieden waren (71\%), für $11 \%$ waren Nebenwirkungen unter der bisherigen Medikation der Grund, etwa $17 \%$ wurden erstmals gegen
RLS behandelt. $50 \%$ der vorbehandelten Teilnehmer hatten L-Dopa erhalten, 86\% der Patienten hatten auch tagsüber RLSBeschweren in Ruhesituationen.

Der Erfolg der Therapie wurde nach etwa drei Monaten anhand der RLS6-Skala überprüft. Sie basiert auf sechs Fragen, bei denen die Patienten die Ausprägung der Symptome mit 1 bis 10 Punkten bewerten müssen. Gefragt wird nach der Zufriedenheit mit dem Schlaf während der vergangenen sieben Nächte, nach den Symptomen beim Einschlafen und in der Nacht sowie tagsüber in Ruhe und in Aktivität. Bewerten sollen die Patienten zudem die Müdigkeit am Tage. Vor Beginn der Therapie lagen die Werte meist zwischen 5 und 6,4 Punkten, sie gingen während der Therapie um 2 bis 3 Punkte zurück.

Insgesamt, so Stiasny-Kolster, ließ sich ein deutlicher Rückgang der RLS-Symptome bei bis zu 70\% der Patienten nach Einstellung auf Rotigotin beobachten, und zwar sowohl während des Einschlafens und nachts als auch tagsüber.

Thomas Müller, Springer Medizin

Satellitensymposium „Idiopathisches Parkinsonund Restless-Legs-Syndrom: Neues aus Klinik und Praxis", 85. DGN-Kongress, Hamburg, 28.9.2012; Veranstalter: UCB Pharma

\section{Orale Therapie der Multiplen Sklerose}

\section{Daten sprechen für die frühe Gabe}

Bei Patienten mit MS lohnt sich ein früher Therapiebeginn mit Fingolimod (Gilenya ${ }^{\circledast}$ ) - sie bleiben damit länger schubfrei als bei einem späteren Beginn. Neue Daten bestätigen zudem eine gute kardiale Sicherheit auch bei Patienten mit Bradykardie und bekanntem AV-Block.

Eine Therapie mit Fingolimod kann die jährliche Schubrate sowohl im Vergleich zu Placebo als auch zu einer Basistherapie mit Interferon beta-1a um mehr als die Hälfte reduzieren - das haben Phase-II- und Phase-III-Studien ergeben, rief Professor Finn Sellebjerg, Kopenhagen, in Erinnerung. Von der Therapie scheinen besonders auch solche Patienten zu profitieren, die trotz Interferon-Behandlung eine hohe Krankheitsaktivität zeigen. So gab es in dieser Gruppe von Patienten in der Zulassungsstudie TRANSFORMS nach einem Jahr unter Fingolimod $61 \%$ weniger Schübe als unter Interferon beta-1a, sagte Sellebjerg beim ENS-Kongress in Prag. Fünfjahresdaten der Stu- 